\title{
Genomic organisation analysis of novel immunoglobulin-like transcripts in Atlantic salmon (Salmo salar) reveals a tightly clustered and multigene family
}

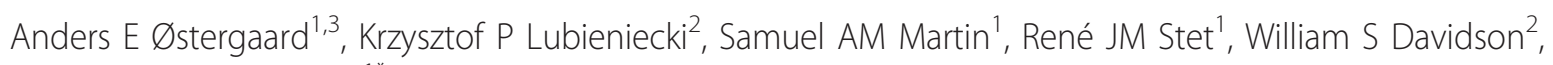
Christopher J Secombes $^{1 *}$

\begin{abstract}
Background: Several novel immunoglobulin-like transcripts (NILTs) which have previously been identified in the salmonid species rainbow trout (Oncorhynchus mykiss) contain either one or two extracellular lg domains of the V-type. NILTs also possess either an immunoreceptor tyrosine-based activating motif (ITAM) or immunoreceptor tyrosine-based inhibitory motifs (ITIMS) in the cytoplasmic region resulting in different signalling abilities. Here we report for the first time the genomic organisation and structure of the multigene family of NILTs in Atlantic salmon (Salmo salar) using a BAC sequencing approach.

Results: We have identified six novel Atlantic salmon NILT genes (Ssa-NILT1-6), two pseudogenes (Ssa-NILTp1 and Ssa-NILTp2) and seven genes encoding putative transposable elements in one BAC covering more than $200 \mathrm{kbp}$. Ssa-NILT1, 2, 4, 5 and 6 contain one Ig domain, all having a $\mathrm{CX}_{3} \mathrm{C}$ motif, whereas Ssa-NILT3 contains two Ig domains, having a $\mathrm{CX}_{6} \mathrm{C}$ motif in $\lg 1$ and a $\mathrm{CX}_{7} \mathrm{C}$ motif in Ig2. Atlantic salmon NILTs possess several ITIMs in the cytoplasmic region and the ITIM-bearing exons are in phase 0 . A comparison of identity between the amino acid sequences of the $\mathrm{CX}_{3} \mathrm{C}$ Ig domains from NILTs varies from $77 \%$ to $96 \%$. Ssa-NILT1, 2, 3 and 4 were all confirmed to be expressed either by their presence in EST databases (Ssa-NILT1) or RT-PCR (Ssa-NILT2, 3, and 4) using CDNA as template. A survey of the repertoire of putative NILT genes in a single individual revealed three novel genes (SsaNILT7-9) represented by the Ig domain, which together with Ig domains from Ssa-NILT1-6 could be divided into different groups based on specific motifs.
\end{abstract}

Conclusions: This report reveals a tightly clustered, multigene NILT family in Atlantic salmon. By screening a highly redundant Atlantic salmon BAC library we have identified and characterised the genomic organisation of six genes encoding NILT receptors. The genes show similar characteristics to NILTs previously identified in rainbow trout, having highly conserved cysteines in the Ig domain and several inhibitory signalling motifs in the cytoplasmic region. In a single individual three unique NILT Ig domain sequences were discovered at the genomic DNA level, which were divided into two different groups based on a four residue motif after the third cysteine. Our results from the BAC screening and analysis on the repertoire of NILT genes in a single individual indicates that many genes of this expanding Ig containing NILT family are still to be discovered in fish.

\footnotetext{
* Correspondence: c.secombes@abdn.ac.uk

${ }^{1}$ Scottish Fish Immunology Research Centre, University of Aberdeen, Zoology

Building, Tillydrone Avenue, Aberdeen AB24 2TZ, UK

Full list of author information is available at the end of the article
} 


\section{Background}

Disease control and health is central to the production of salmon in aquaculture and more knowledge of the immune system in fish might help prevent infectious disease outbreaks. Salmon inhabit a temperate environment and their adaptive immune system is not as rapid as in mammals. Therefore, they rely to a greater degree on the innate immune system to combat pathogens [1]. A wide range of activating and inhibitory receptors play a role in the innate immune system, of which many are expressed on neutrophils, macrophages and natural killer (NK) cells. These receptors recognise conserved pathogen associated molecular patterns (PAMPs) released from or found on the surface of pathogens [2], and result in the activation of responsive cells. Many of these cell-surface receptors contain immunoglobulin-like (Ig) domains [3] and several genes have been found in clusters such as the leukocyte receptor complex (LRC) [4] and the triggering receptor expressed on myeloid cells (TREM) cluster [5] in mammals. The LRC is a very gene dense region, spanning $1 \mathrm{Mb}$, which includes the killer cell Ig-like receptors (KIRs), leukocyte Ig-like receptors (LILRs) and the natural cytotoxicity receptor (NCR) NKp46 [6]. The TREM cluster on human chromosome 6 harbours genes such as TREM1 and 2, as well as the NCR named NKp44 [5,7]. TREM receptors are involved in the amplification and attenuation of the inflammatory response [8,9], while the NKp44 receptor activates NK cells [10]. All these receptors are type I transmembrane proteins characterised by the presence of a variable number of extra-cellular Ig domains of either the C2-type or the novel V-type [10,11]. The Ig domains are usually followed by a connecting peptide, a transmembrane region, and a cytoplasmic tail. Some inhibitory receptors have a long cytoplasmic region containing one or more immunoreceptor tyrosine-based inhibitory motifs (ITIMs) [12], which block NK cellmediated cytotoxicity $[13,14]$. The cytoplasmic regions of activating receptors are short and associate with

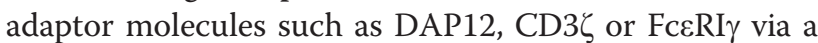
positively charged residue (arginine or lysine) in their transmembrane region. These adaptor proteins contain a negatively charged residue in their transmembrane region and an immunoreceptor tyrosine-based activating motif (ITAM) in their cytoplasmic region [15].

In several species of teleost fish, receptors belonging to the Ig super family (IgSF) have been reported. These include the novel immune-type receptors (NITRs) reported in Southern pufferfish [16], zebrafish [17], channel catfish [18], rainbow trout [19], Japanese flounder [20] and sea bass [21], and the novel immunoglobulin-like transcript (NILT) genes described in carp [22] and rainbow trout $[23,24]$. Most NITRs possess a V-type
Ig domain and most of them also have a second Ig domain of the V/C2-type followed by a transmembrane and cytoplasmic region. The majority of NITRs contain an ITIM, whereas a few contain an ITAM instead.

NILT receptors have either one or two extracellular Ig domains, a connecting peptide, a transmembrane region, and a cytoplasmic region containing the signalling motifs, and are expressed mainly in lymphoid tissues $[23,24]$. Homology modelling has indicated that these receptors have an extra-cellular Ig domain structurally similar to the V-type Ig domain of human NKp44. In carp, the NILT genes were shown to belong to a multigene family and analysis of the NILT Ig-encoding sequences revealed extensive haplotypic and allelic polymorphism [22]. Several NILT genes have been characterized in rainbow trout [24] suggesting the multigene status of NILT in salmonids. These observations prompted us to investigate the genomic organization and structure of NILT genes in Atlantic salmon using a bacterial artificial chromosome (BAC) library. This paper describes the first study on NILT in Atlantic salmon and reveals the genomic organization of several NILT genes in a single BAC clone sequence. In addition, the analysis shows that these NILT genes are tightly clustered in Atlantic salmon, with Ig domains structurally related to the novel V-type Ig domain of NKp44 and containing inhibitory signalling motifs.

\section{Results}

Identification and gene organization of NILTs in Atlantic salmon

Two partial NILT sequences [GenBank:DW550613, GenBank:DW564289] [25], from Atlantic salmon were used to design a 40-mer oligonucleotide probe located in the Ig domain. The probe hybridised to several BAC clones located within contig 341 in the physical map of the Atlantic salmon genome generated based on HindIII fingerprinting (Figure 1) [26], http://www.asalbase.org, of which one (S0024B13) was selected for shotgun sequencing. A continuous sequence of 149,704 bp was obtained after shotgun sequencing, contig assembling and gap closing using specific primers located in the contig ends [GenBank:GU552297]. Examination of this region (contig 4) identified four full-length NILT genes (Ssa-NILT1, Ssa-NILT2, Ssa-NILT3 and Ssa-NILT4) and one partial NILT gene (Ssa-NILT5). In the neighbouring contig 3 (size $35,964 \mathrm{bp}$ ) the putative $5^{\prime}$ end of $S s a$ NILT5 was identified as well as a predicted Ssa-NILT6. The NILT genes are orientated in both 3' and 5' directions in the BAC clone sequence (Figure 2). The open reading frames (ORF) of the genes containing one Ig domain are; Ssa-NILT1 (1032 bp), Ssa-NILT2 (993 bp), Ssa-NILT4 (1062 bp), Ssa-NILT5 (933 bp) and 


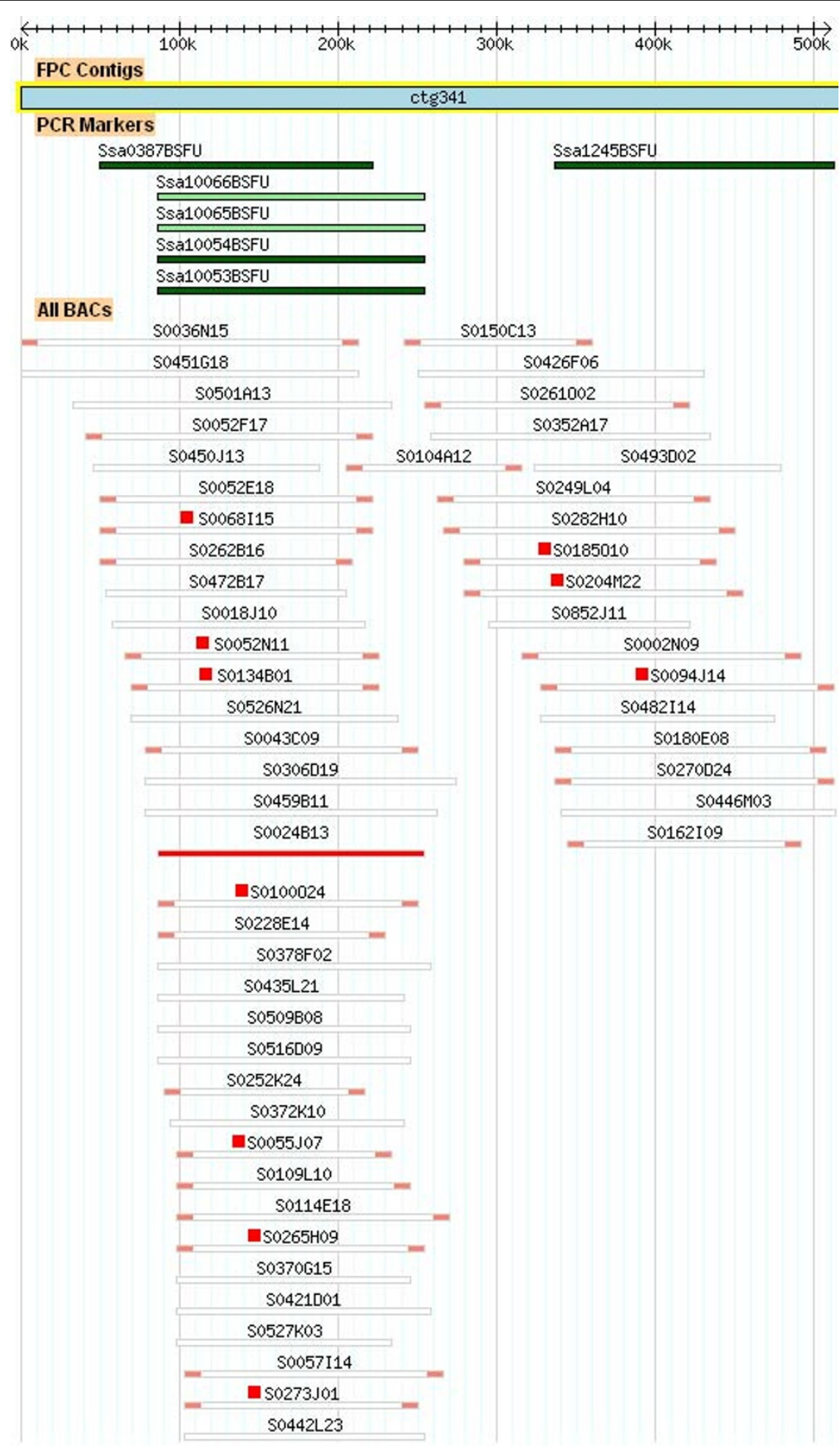

Figure 1 Screenshot of FPC contig 341 adapted from http://www.asalbase.org. BAC clone S0024B13 was chosen for shotgun sequencing (indicated in red). BAC clones positive for NILT hybridization probe are indicated by a red square. The position of PCR markers are highlighted with dark and light green bars. All BAC clones have been end sequenced (indicated in light red). 
a

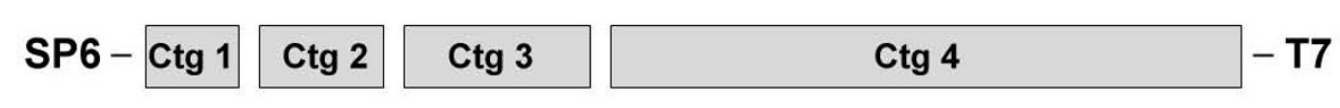

b

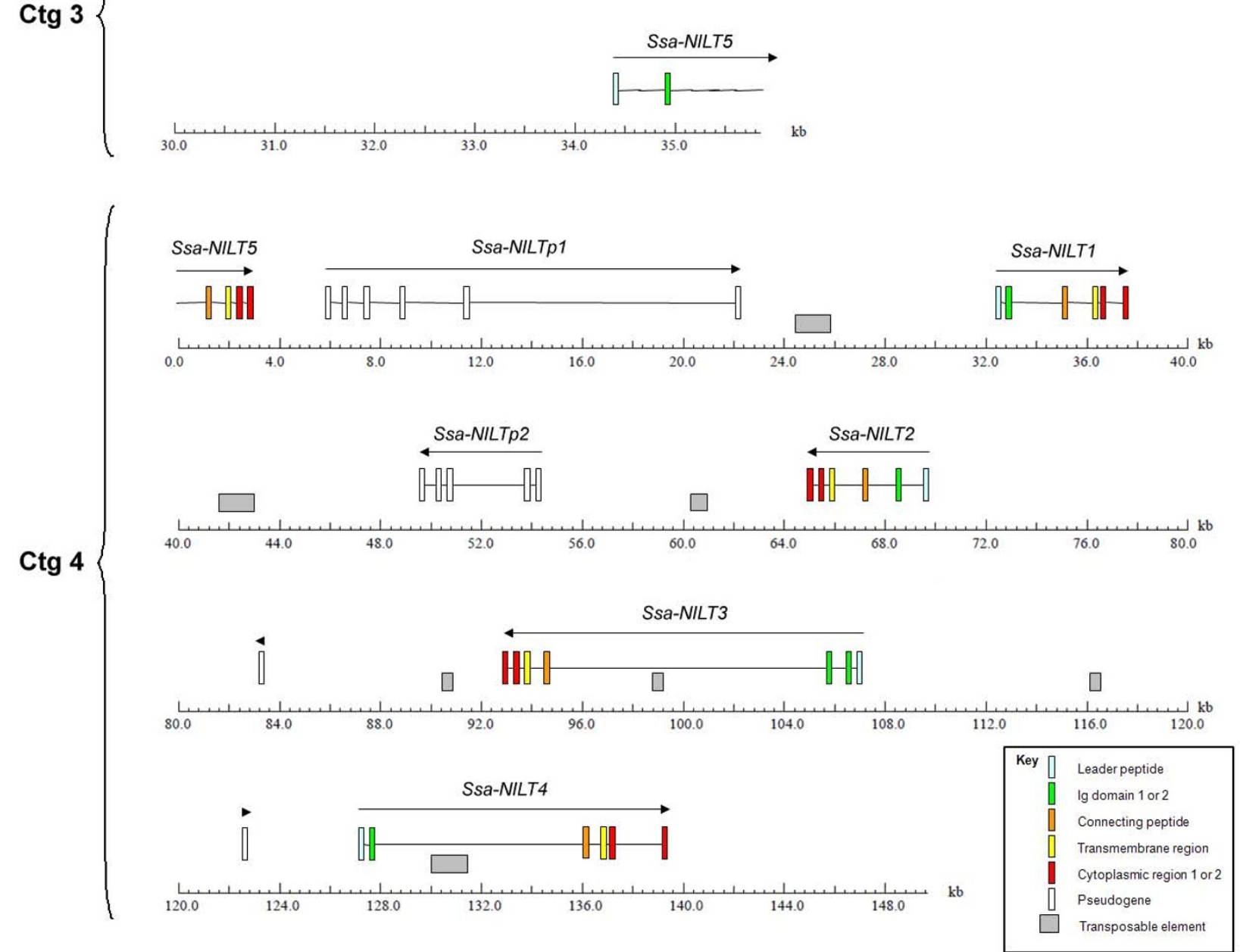

Figure 2 Assembly of NILT-containing BAC clone. a. Graphic illustration of the orientation and the number of contigs (ctg) after assembly. b. Genomic organization of the Atlantic salmon (Ssa) NILT genes identified in BAC clone S0024B13. Contig 3 contains a partial and a full-length predicted NILT. Contig 4 contains four full-length NILT genes, one partial NILT gene and two NILT pseudogenes.

Ssa-NILT6 (945 bp) with each gene consisting of six exons and five introns. The Ig domain, connecting peptide and transmembrane region are encoded in exons 2, 3 , and 4, respectively. Exon 4 also contains part of the cytoplasmic region, while the remainder of the cytoplasmic region and the 3' untranslated region are encoded in exons 5 (Cyt1) and 6 (Cyt2). Introns 1-4 are in phase 1 , whereas intron 5 is in phase 0 (Figure 3). The Ssa-NILT3 (1278 bp) gene encodes the only NILT discovered so far in Atlantic salmon to have two Ig 


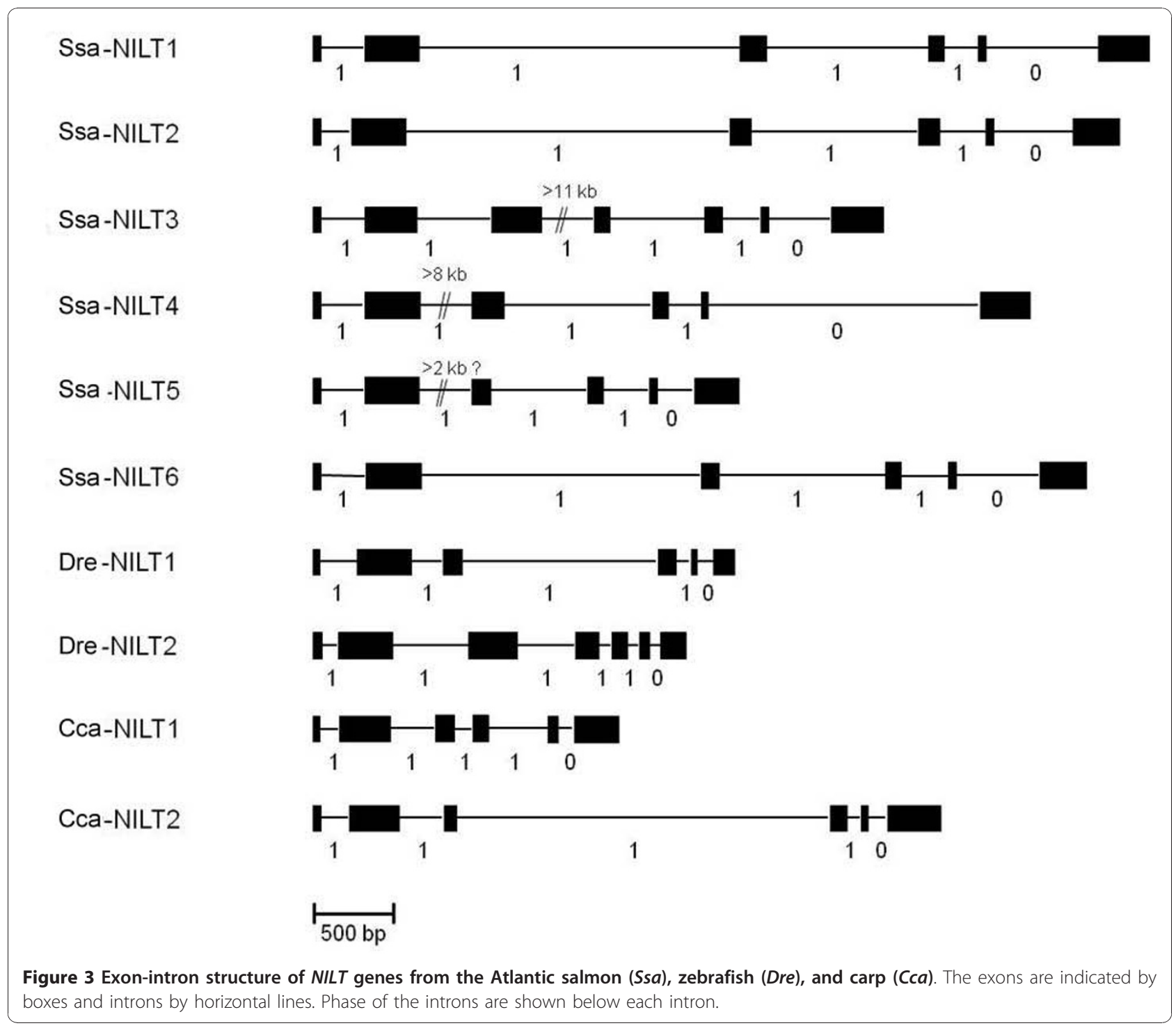

domains, and containing an additional intron in phase 1. The phases of the introns are highly conserved.

The Ig domains of all the Ssa-NILTs are of the V-type and contain the characteristic cysteines at positions 17 and 89 responsible for disulphide bridge formation (Figure 4). Additional cysteines separated by three amino acids $\left(\mathrm{CX}_{3} \mathrm{C}\right)$ are present in the Ig domains of Ssa-NILT1, Ssa-NILT2, Ssa-NILT4, Ssa-NILT5 and SsaNILT6 whereas they are separated by six amino acids $\left(\mathrm{CX}_{6} \mathrm{C}\right)$ in the Ig1 domain of Ssa-NILT3. In the second Ig domain of Ssa-NILT3 they are separated by seven amino acids $\left(\mathrm{CX}_{7} \mathrm{C}\right)$. These cysteines may form a second disulphide bridge. The connecting peptide of Ssa-NILT1 contains a high number of serine/threonine residues, 29/56 respectively. Twenty-three serine residues were identified using the NetPhos server 2.0 http://www.cbs. $\mathrm{dtu} . \mathrm{dk} /$ services/NetPhos/ in the connecting peptide of
Ssa-NILT1 to be potential phophorylation sites (data not shown). This high proportion of serine and threonine residues is also observed in the connecting peptide of Ssa-NILT2-6 with 22/45, 15/33, 36/68, 22/38 and 20/ 37 residues, respectively (Figure 4). In addition, the Shannon entropy ( $H$; http://imed.med.ucm.es/PVS/) was calculated for the Ssa-NILT sequences. Positions with $H$ values above 1.3 are considered to be variable, while those with $H$ values smaller than 1.3 are identified as conserved. The Shannon plot revealed that amino acid positions in the Ig domain and in the connecting peptide of the deduced NILT proteins could be considered as variable (Figure 4). The cytoplasmic regions of NILTs in Atlantic salmon range in size from 332 to 377 residues and the percentage of identity between the NILTs varies from $63 \%$ to $84 \%$ at the amino acid level. The NILTs have either four or five inhibitory motifs in the 


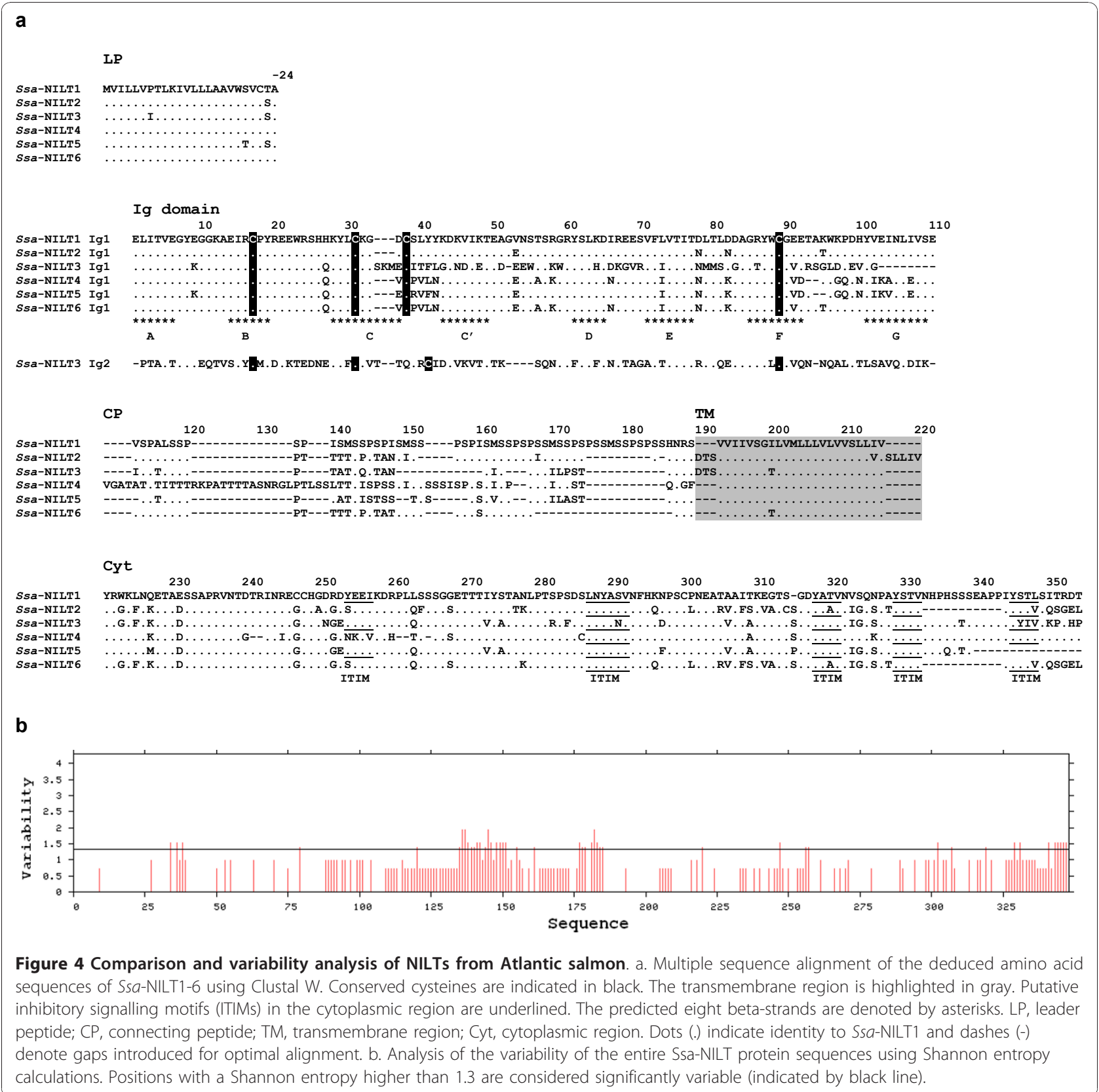

cytoplasmic region, which adhere to the consensus of ITIMs (S/I/V/LxYxxI/V/L or YxxI/V/L) (Figure 4). These correspond to the NILTs found in rainbow trout, which also contain several inhibitory signalling motifs [24]. Ssa-NILT1, Ssa-NILT2, Ssa-NILT3, and Ssa-NILT4 were all confirmed to be expressed either by their presence in EST databases http://web.uvic.ca/grasp (SsaNILT1) or RT-PCR (Ssa-NILT2, Ssa-NILT3, and SsaNILT4) using cDNA as template and primers listed in Table 1 . We were not able amplify a specific product representing Ssa-NILT5, while a product for Ssa-NILT6
Table 1 Primer sequences for amplifying full-length SsaNILT2, 3 and 4, and Ig domains

\begin{tabular}{lll}
\hline Primers & Sequence $\left(\mathbf{5}^{\prime}\right.$-3') & Amplifying \\
\hline Ssa-NILT-f1 & ACTAGCTGGGAGCCACAAGTCATC & Ssa-NILT2/Ssa-NILT4 \\
Ssa-NILT-f2 & CATCATGGTATCTTGCTGGTCAT & Ssa-NILT3 \\
Ssa-NILT-r1 & TTGACGTTGGCGTAGTTGAG & Ssa-NILT2/Ssa-NILT3 \\
Ssa-NILT-r2 & GTCCCTTATGATTGGTTGTCAGTG & Ssa-NILT4 \\
Ssa-panlg-f & GAGTTGATCACAGTGGAAGGA & Pan-NILT Ig \\
Ssa-panlg-r & TCCRCACCAGTATSTYCCAGC & Pan-NILT Ig \\
\hline
\end{tabular}


was amplified using genomic DNA as template. However, the primers used for amplifying a specific product representing Ssa-NILT6 were very difficult to design due to high similarity between NILT genes in the BAC sequence.

A pseudogene, Ssa-NILTp1, was identified between nucleotide position 6197 and 22738 in the BAC clone sequence (contig 4), comprising a leader peptide, two putative Ig domains, a connecting peptide, a transmembrane region and part of the cytoplasmic region (Figure 2). The pseudo gene contained a stop codon at nucleotide position 7013 in the Ig1 domain immediately after the last cysteine. Another pseudogene, Ssa-NILTp2, orientated in the reverse direction was identified between nucleotide position 49770 and 54621 in the same contig. Ssa-NILTp2 consists of a leader peptide and one Ig domain followed by the initial part of a second Ig domain, a transmembrane region and the cytoplasmic tail. An exon coding for the connecting peptide was not identified suggesting that it may not function as a true NILT. Two additional Ig domains, nucleotide position 83454 to 83746 and 122661 to 122780 were also discovered in contig 31 , both of them containing either a stop codon or a nucleotide deletion interrupting the reading frame.

Intron 3 from Ssa-NILT3 is large (>11 kbp) and contained a conserved domain belonging to the reverse transcriptase (RT) superfamily. GENSCAN predictions [27] and BLASTn [28] searches of the non-redundant nucleotide database using the BAC clone sequence as query predicted seven transposable elements (Figure 2) two of which belong to the piggyBac-like DNA transposon family and one belongs to the Tc1-like transposon family [29]. The remaining four transposable elements contained conserved domains belonging to the RT superfamily.

A phylogenetic tree was constructed using the amino acid sequence of the Ig domains of NILTs from Atlantic salmon, rainbow trout, carp and zebrafish together with human Ig receptors (Figure 5). It shows a clear division of Ig1 and Ig2 domains as well as clustering of salmonids and cyprinids.

\section{NILT repertoire in Atlantic salmon}

Genomic DNA was extracted from a single individual and used as template in ten independent PCRs in order to amplify a specific part of the Ig domain. Ten clones from each PCR were sequenced and only NILT sequences observed more than once were included in the analysis of the repertoire. Eighty one clones were found positive for NILT resulting in seven different sequences and 19 singletons at the amino acid level. Despite using degenerate oligonucleotide primers designed to amplify Ig domains containing either $\mathrm{CX}_{3} \mathrm{C}$ or $\mathrm{CX}_{6} \mathrm{C}$ motifs (Table 1 ) only Ig domains containing $\mathrm{CX}_{3} \mathrm{C}$ motifs were observed.

Of the seven different sequences retrieved, four were identical to either Ssa-NILT1,Ssa-NILT2, Ssa-NILT4 or Ssa-NILT6 at the nucleotide level, leaving three novel Ig domains of NILTs Ssa-NILT7-9. Multiple alignment of the amino acid sequences and subsequent phylogenetic analysis revealed a division of NILT Ig domains into two different types (Figure 6). Group 1, comprising SsaNILT1, Ssa-NILT2, and Ssa-NILT8, have Ig domains containing a $\mathrm{CX}_{3} \mathrm{C}$ motif as well as a histidine residue at position 20, a SLYY motif located at positions 32 to 35 (immediately after the third cysteine), and a leucine residue at position 66. Group 2, comprising Ssa-NILT4, SsaNILT5, Ssa-NILT6, Ssa-NILT7, and Ssa-NILT9, have Ig domains also containing a $\mathrm{CX}_{3} \mathrm{C}$ motif, but having a glutamine residue at position 20 , a $(\mathrm{P} / \mathrm{R}) \mathrm{V}(\mathrm{F} / \mathrm{L}) \mathrm{N}$ motif located from position 32 to 35 , and an isoleucine residue at position 66. In the Ig1 domain of Ssa-NILT3, which contains a $\mathrm{CX}_{6} \mathrm{C}$ motif, there was a characteristic glutamine residue at position 20 and the isoleucine residue in position 66 . The Ig2 domain, containing a $\mathrm{CX}_{7} \mathrm{C}$ motif, is significantly different and was included in the phylogenetic tree as an out-group.

\section{Discussion}

Pattern recognition receptor (PRR) genes encoding the tertiary structure of an Ig domain belong to the immunoglobulin superfamily. These Ig containing receptors are found throughout the animal kingdom and several clusters have been identified in mammals [30]. A classic example is the leukocyte receptor complex, which contains several gene families encoding PRRs having single or multiple Ig domains [6]. It is hypothesised that they have evolved through duplication to give a wide array of Ig-containing PRRs within the innate immune system of vertebrates in order to recognise potential pathogenic organisms. In this paper we describe for the first time the characterisation and genomic organisation of the Igcontaining receptor termed novel immunoglobulin-like transcript (NILT) in the Atlantic salmon.

Six NILT genes, equally distributed within a BAC sequence (S0024B13), were identified. In addition, two pseudogenes as well as two single pseudo Ig domains and seven transposable elements were identified. Comparison of the genomic organisation revealed a similar structure for the NILT genes, with the exception of SsaNILT3, which contains an additional Ig domain (Ig2) encoded by exon 2 a followed by a long intron 2 a of 11 kbp (Figure 2). The occurrence of a NILT with two Ig domains was also present in rainbow trout [24], in which the intron following the Ig2 encoding exon in Omy-NILT1 has a size of $5.8 \mathrm{~kb}$ (Table 2) [23]. 


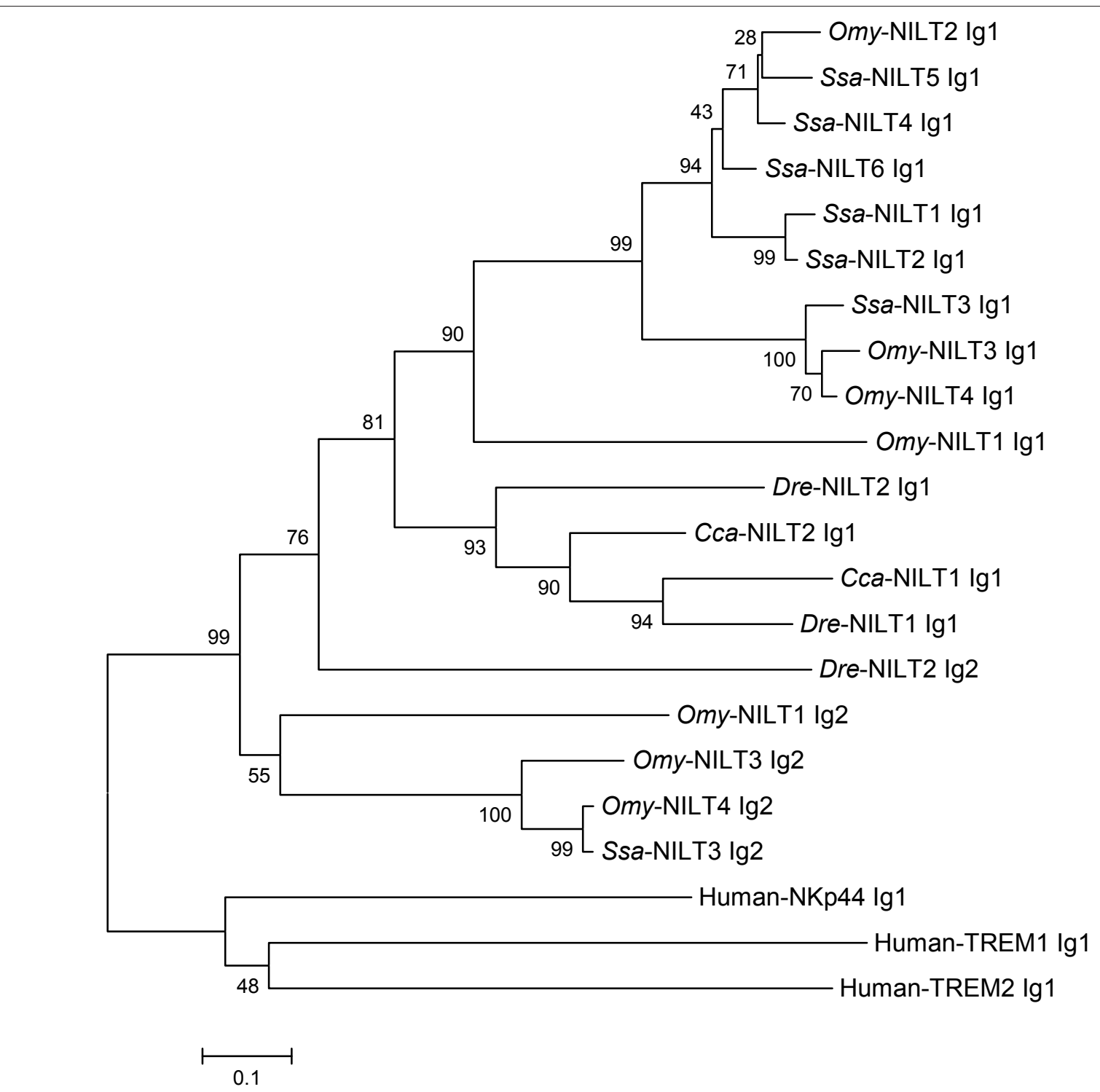

Figure 5 Phylogenetic tree showing the relationship between NILTs from Atlantic salmon, rainbow trout, carp and zebrafish and NKp44 and TREM molecules from human using the amino acid sequences of the Ig domains. The unrooted tree was built by the neighbour-joining method using Clustal W and the MEGA 4 packages and bootstrapped 10,000 times. The scale bar corresponds to 0.1 estimated amino acid substitutions per site. [GenBank: Omy-NILT1 [23]; Omy-NILT2, FM180056; Omy-NILT3, FM180057; Omy-NILT4, FM180058; CCa-NILT1, CAH19212; CCa-NILT2, CAH19213; Dre-NILT1, BN001234; Dre-NILT2, BN001235; Human-TREM1, AAL74018; Human-TREM2, AAH32362; Human-NKp44, AJ225109].

However, when examining the equivalent intron in DreNILT1 predicted from zebrafish, it was shown to be only 362 bp long [24] indicating a more compact gene structure of NILT genes in cyprinids compared to salmonids. Intron 1 in all Atlantic salmon NILTs, except for Ssa-NILT2, has the exact same size of 275 nucleotides and showed $>96 \%$ identity. The remaining introns differ in length, however, intron 2 and 3 from SsaNILT1 and Ssa-NILT2 also revealed a high percentage of identity (>95\%). Between different teleost species the sizes of introns and exons are generally highly conserved
(Table 2), implying a close relationship of NILT genes across species. Comparison of the amino acid sequences of the Ig encoding exons from Ssa-NILT1 and SsaNILT2 revealed a strikingly high percentage of identity (96\%) (Table 3). In contrast, the percent identity between the cytoplasmic region encoded by exon 5 and 6 of Ssa-NILT1 and Ssa-NILT2 was only $67 \%$. The lowest percent of identity between Atlantic salmon Ig domains was seen when comparing Ig1 and Ig2 in SsaNILT3 (26\%), which might indicate that NILT receptors with two Ig domains have a larger repertoire of binding 

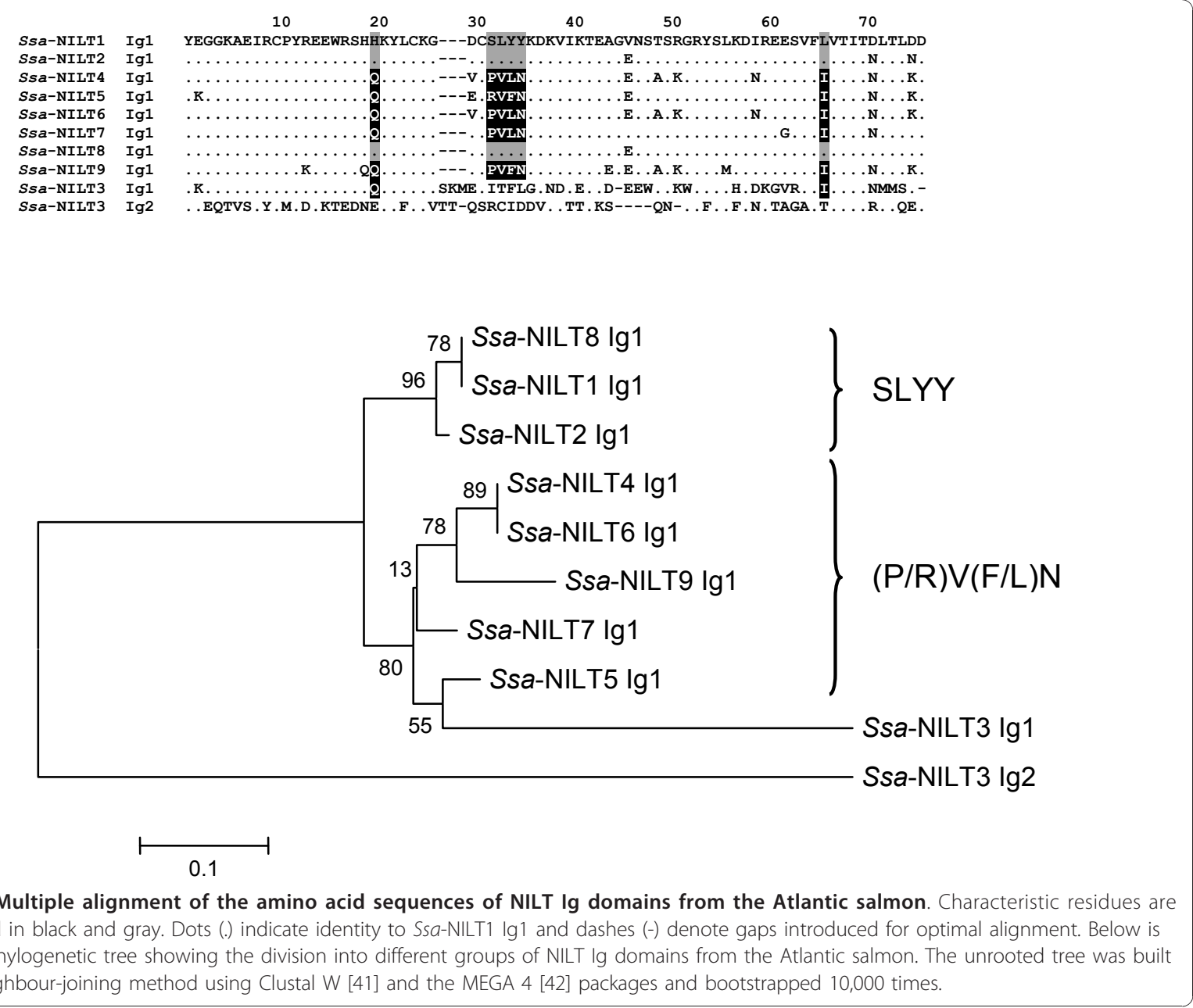

specificities than NILT receptors with one Ig domain. So far no ligands for the NILT receptor have been identified, but future work will determine if there is any resemblance to the highly polymorphic KIRs in humans, which bind another highly polymorphic ligand, namely major histocompatibility complex (MHC) class I [31].

Two pseudogenes, Ssa-NILTp1 and Ssa-NILTp2, were identified in the BAC clone sequence as well as two separate pseudogenes each encoding a single Ig domain. The two pseudogenes have many features in common with Ssa-NILT1-6, but Ssa-NILTp1 contains a stop codon in the Ig1 domain and exon 5 was absent. SsaNILTp 2 contains an Ig1 domain highly similar to the Ig1 domain of Ssa-NILT3 (99\%). The initial part of a second Ig domain was also identified. However, immediately after the first cysteine in the Ig2 domain encoding exon, a nucleotide deletion results in a shift of reading frame. In addition, exon 3 encoding the connecting peptide was absent.

All NILTs identified in the BAC clone sequence contain several ITIM signalling motifs in the cytoplasmic region encoded by exon 6 . Exons bearing ITIM sequences are always in phase 0 , i.e. the intron-exon boundary does not interrupt a codon [32]. In addition, many genes belonging to the immunoglobulin superfamily have exons encoding the Ig domain that are in phase 1 [33], which is also observed in the NILT genes from the Atlantic salmon (Figure 3). Having an ITIM-containing exon in phase 0 implies that the signalling properties of the Ssa-NILT receptors are functional and phosphorylation of the tyrosine residues in the ITIM motif leading to inhibition is most likely to occur.

The BAC clone analysis resulted in the identification of several NILTs, which suggests the presence of more than six NILTs in Atlantic salmon. We carried out a study using genomic DNA and primers located in conserved regions of the Ig domain. By using this approach we obtained three novel NILT Ig sequences (Ssa-NILT79) and 19 singletons at the amino acid level out of 81 clones. This implies that NILT genes in Atlantic salmon are a multigene family of related genes. Interestingly, none of the 81 clones contained the $\mathrm{CX}_{6} \mathrm{C}$ motif, which 
Table 2 Nucleotide lengths of exons and introns of NILTs found in teleost fish

\begin{tabular}{|c|c|c|c|c|c|c|c|c|c|c|c|c|c|c|c|}
\hline & $\begin{array}{r}\text { LP } \\
\text { Exon } 1 \\
\end{array}$ & Intron 1 & $\lg 1$ Exon 2 & Intron 2 & $\lg 2$ Exon 2a & Intron $2 a$ & CP Exon 3 & Intron 3 & TM Exon 4 & Intron 4 & Cyt1 Exon 5 & Intron 5 & Cyt2 Exon 6 & 3'UTR & Signalling motif \\
\hline Ssa-NILT1 & 46 & 275 & 348 & 2056 & - & - & 168 & 1074 & 96 & 218 & 50 & 753 & 324 & 395 & $5 \mathrm{ITIMs}$ \\
\hline Ssa-NILT2 & 46 & 180 & 348 & 2067 & - & - & 135 & 1063 & 120 & 286 & 50 & 511 & 294 & 2232 & 4 ITIMs \\
\hline Ssa-NILT3 & 46 & 275 & 330 & 469 & 321 & 11068 & 99 & 610 & 105 & 242 & 50 & 410 & 327 & 687 & 5 ITIMs \\
\hline Ssa-NILT4 & 46 & 275 & 354 & 8112 & - & - & 204 & 941 & 96 & 212 & 44 & 1728 & 318 & 1181 & 4 ITIMs \\
\hline Ssa-NILT5 & 46 & 275 & 342 & $>2381$ & - & - & 117 & 619 & 96 & 288 & 50 & 227 & 282 & 443 & 4 ITIMs \\
\hline Ssa-NILT6 & 46 & 275 & 348 & 1789 & - & - & 111 & 1072 & 96 & 308 & 50 & 526 & 294 & 2230 & 4 ITIMs \\
\hline $\begin{array}{l}\text { Omy- } \\
\text { NILT1 }\end{array}$ & 88 & 182 & 336 & 1061 & 318 & - & - & - & - & - & - & - & - & 162 & ITIM/ITAM \\
\hline $\begin{array}{l}\text { Omy- } \\
\text { NILT1 }{ }^{L}\end{array}$ & 88 & 182 & 336 & 1061 & 318 & $\sim 5800$ & 141 & - & 69 & - & - & - & 417 & 404 & ITIM/ITAM \\
\hline $\begin{array}{l}\text { Omy- } \\
\text { NILT2 }\end{array}$ & 46 & N.D. & 342 & N.D. & - & - & 132 & N.D. & 90 & N.D. & 44 & N.D. & 135 & *391 & 1 ITAM \\
\hline $\begin{array}{l}\text { Omy- } \\
\text { NILT3 }\end{array}$ & 46 & N.D. & 330 & N.D. & 339 & N.D. & 99 & N.D. & 105 & N.D. & 50 & N.D. & 330 & *83 & 3 ITIMs \\
\hline $\begin{array}{l}\text { Omy- } \\
\text { NILT4 }\end{array}$ & 46 & N.D. & 330 & N.D. & - & N.D. & 117 & N.D. & 105 & N.D. & 50 & N.D. & 327 & 549 & 4 ITIMs \\
\hline $\begin{array}{l}\text { Omy- } \\
\text { NILT4 }\end{array}$ & 46 & N.D. & 330 & N.D. & 321 & N.D. & 117 & N.D. & 105 & N.D. & 50 & N.D. & 327 & 549 & 4 ITIMs \\
\hline Dre-NILT1 & 40 & 241 & 345 & 197 & & - & 117 & 1243 & 117 & 82 & 35 & 103 & 132 & 214 & 1 ITIM \\
\hline Dre-NILT2 & 58 & 91 & 342 & 485 & 309 & 362 & 150 & 80 & 96 & 67 & 62 & 82 & 159 & 509 & 1 ITIM \\
\hline CCa-NILT1 & 40 & 117 & 324 & 279 & - & - & 117 & 112 & 96 & 381 & 59 & 91 & 288 & 206 & 1 ITAM \\
\hline Cca-NILT2 & 49 & 169 & 321 & 276 & - & - & 81 & 2381 & 105 & 88 & 35 & 110 & 339 & 270 & 4 ITIMs \\
\hline
\end{tabular}

Number and type of putative signalling motifs in each NILT are indicated.

(* denotes no poly-A signal observed, N.D. denotes not determined). 
Table 3 Comparison by pairwise alignments (Clustal W) of the NILT Ig domains in Atlantic salmon

\begin{tabular}{llllclll}
\hline & \multicolumn{7}{c}{ Identity } \\
\hline Ssa-NILT1 Ig1 & & 96 & 58 & 40 & 77 & 79 & 85 \\
Ssa-NILT2 Ig1 & 98 & & 59 & 42 & 78 & 81 & 88 \\
Ssa-NILT3 Ig1 & 69 & 70 & & 32 & 63 & 67 & 62 \\
Ssa-NILT3 Ig2 & 48 & 48 & 52 & & 44 & 38 & 44 \\
Ssa-NILT4 Ig1 & 84 & 84 & 71 & 52 & & 91 & 89 \\
Ssa-NILT5 Ig1 & 89 & 89 & 72 & 50 & 93 & & 84 \\
Ssa-NILT6 Ig1 & 89 & 91 & 69 & 53 & 93 & 90 & \\
& & \multicolumn{7}{c}{ Similarity } \\
\end{tabular}

The table shows comparison of $\%$ amino acid identity and similarity.

suggests that a higher number of genes encoding Ig domains containing the $\mathrm{CX}_{3} \mathrm{C}$ motif may be present in the genome. Multiple alignment and phylogenetic analysis divided the $\mathrm{CX}_{3} \mathrm{C}$ sequences into two groups, having different motifs located immediately after the third cysteine (Figure 6). Group 1 molecules contained sequences with a SLYY motif and a histidine and leucine residue at positions 20 and 66, respectively. In contrast, group 2 molecules contained sequences with a $(\mathrm{P} / \mathrm{R}) \mathrm{V}$ $(\mathrm{F} / \mathrm{L}) \mathrm{N}$ motif and a glutamine and isoleucine at positions 20 and 66, respectively. The four residue motifs are located in a loop region between beta-strands $\mathrm{C}$ and $\mathrm{C}^{\prime}$, when comparing the amino acid sequence to the sequence of NKp44 [7]. This observation corresponds to the theory that most variation is detected in loopregions. Future studies will determine if these motifs, as well as the general allelic polymorphism of NILT genes seen in a single individual may contribute to different binding specificities for an as yet unknown ligand. Until now only a few ligands have been identified for nonrearranging receptors with the most investigated being the interaction of classical and non-classical MHC class I molecules to KIRs [34]. NILTs have not been identified in any vertebrates other then teleost to date suggesting that this multigene family of receptors are involved in the control of immune responses in fish only and may represent an ancient type of receptor that has expanded in the fish lineage.

\section{Conclusions}

In conclusion, this report reveals a tightly clustered, multigene family of novel immunoglobulin-like transcripts (NILT) in Atlantic salmon. By screening a highly redundant Atlantic salmon BAC library we have identified and characterised the genomic organisation of six genes encoding NILT receptors. The genes show similar characteristics to NILTs previously identified in rainbow trout [24] having highly conserved cysteines in the Ig domain and several inhibitory signalling motifs in the cytoplasmic region. In a single individual, three additional and NILT sequences were discovered at the genomic DNA level, and allowed division of NILTs into two different groups based on a four residue motif after the third cysteine in the Ig domain. Together with the NILTs from the BAC clone sequence we now know that multiple NILT genes exist in teleost fish and it will be interesting to discover if and how they differ functionally.

\section{Methods \\ Screening the Atlantic salmon CHORI-214 BAC library for NILT genes}

The Atlantic salmon bacterial artificial chromosome (BAC) library, CHORI-214, was obtained from BACPAC Resources, Children's Hospital Oakland Research Institute (CHORI), Oakland, CA. The average insert size of the library is $188 \mathrm{kbp}$, representing 18 -fold genome coverage [35]. In preparation for screening the library, the filters were prehybridised for $1 \mathrm{~h}$ at $65^{\circ} \mathrm{C}$ in a solution consisting of $5 \times$ SSC, $5 \times$ Denhardt's solution, and $0.5 \%$ SDS. The $40-$ mer oligonucleotide probe (5' GGAGTGGACAAATGGGGACAAGATAACTACATTAATA3') used for screening the library was labelled at the $5^{\prime}$ end with $\gamma_{-}{ }^{32}$ P-ATP using $\mathrm{T} 4$ polynucleotide kinase (Invitrogen) in a $10 \mu \mathrm{l}$ volume reaction and incubated for at least $30 \mathrm{~min}$ at $37 \mathrm{C}$ before it was added to the hybridisation solution. The labelling reaction included $1 \mu \mathrm{l}$ of $10 \mu \mathrm{M}$ probe, $10 \mathrm{U}$ of T4 polynucleotide kinase, $2 \mu \mathrm{l}$ of $5 \times$ forward reaction buffer, $2 \mu \mathrm{l}$ of $\gamma-{ }_{-}^{32}$ P-ATP $(3000 \mathrm{Ci} / \mathrm{mmol})$ and $4 \mu \mathrm{l}$ of water. The hybridisation solution was as above and to each prehybridised filter $1.6 \mu \mathrm{l}$ of radioactively labelled oligonucleotide probe were added and the hybridisation was carried out at $65^{\circ} \mathrm{C}$ overnight $(\sim 18 \mathrm{~h})$. Following hybridisation, the filters were washed three times in $1 \times \mathrm{SSC}$ and $0.1 \%$ SDS at $50^{\circ} \mathrm{C}$ for $1 \mathrm{~h}$ for each wash. The washed filters were exposed to phosphor screens overnight and scanned using the Typhoon 9410 Phosphor Imager (Amersham Biosciences). The BAC clones positive by hybridisation were picked from the library and grown in $5 \mathrm{ml}$ of LB medium containing $20 \mu \mathrm{g} /$ $\mathrm{ml}$ chloramphenicol for $14-16 \mathrm{~h}$ at $37^{\circ} \mathrm{C}$ with shaking at 250-300 rpm. PCR amplification of the hybridisationpositive clones was performed in a Thermal Cycler (Biometra) using NILT specific primers (Ss-NILT-f: GCAGAGAT CAGATGCCCCTA, Ss-NILT-r: ATCTTGTCCCCATTTG TCCA) to determine if these BACs contain sequences corresponding to the NILT gene. All PCR amplifications were performed in a $25 \mu \mathrm{l}$ reaction volume using the following conditions: initial denaturation at $95^{\circ} \mathrm{C}$ for 5 min followed by 35 cycles at $95^{\circ} \mathrm{C}$ for $45 \mathrm{~s}, 65^{\circ} \mathrm{C}$ for $45 \mathrm{~s}, 72^{\circ} \mathrm{C}$ for $45 \mathrm{~s}$ and finally one cycle at $72^{\circ} \mathrm{C}$ for $5 \mathrm{~min}$. For each DNA amplification, $0.05 \mathrm{U}$ Taq DNA polymerase, 12.5 pmoles of each primer, $1 \times$ PCR buffer containing $1.5 \mathrm{mM} \mathrm{MgCl}_{2}$, $50 \mu \mathrm{M}$ of dNTPs and $25 \mathrm{ng}$ of BAC DNA template were used. The amplification products were analysed by 
electrophoresis through a $1.5 \%$ agarose gel containing $1 \times$ TBE and $0.5 \mu \mathrm{g} / \mathrm{ml}$ ethidium bromide and visualised using a UV trans-illuminator (Ultra-Violet Products).

\section{Shotgun sequencing, assembly and annotation of the BAC S0024B13}

Isolation of the BAC DNA and shotgun library construction were carried out as described by Johnstone et al. [36]. Briefly, the isolated BAC DNA was sheared by sonication, blunt-end repaired using the End-It $\mathrm{t}^{\mathrm{m}} \mathrm{DNA}$ End-Repair Kit (Epicentre) and size fractionated by agarose gel electrophoresis. The region containing 2 to $5 \mathrm{~kb}$ fragments was excised and gel purified using a Gel Purification Kit (Qiagen) following the manufacturer's instructions. The fragments were ligated into pUC19 plasmid, which had been cut with SmaI and treated with shrimp alkaline phosphatase, and then used to transform E. coli XL1 Blue supercompetent cells (Stratagene). Large-scale colony picking was performed using a QPix-2 colony picker (Genetix) and 2,304 clones were sent for sequencing at the Michael Smith Genome Sciences Centre (Vancouver). Base calling and assembling were done using Phred and Phrap [37,38]. Assembled sequences were viewed and edited using Consed [39].

GENSCAN [27] was used to predict novel genes and to identify open reading frames. The predicted gene sequences were analysed for the presence of a leader peptide using SignalP 3.0 [40] and transmembrane regions using the TMHMM server $2.0 \mathrm{http}$ ://www.cbs. dtu.dk/services/TMHMM-2.0. Identity as well as similarity with other known sequences was determined using BLAST-n and BLAST-p [28]. Comparisons between sequences were performed with Clustal W [41] and phylogenetic and molecular evolutionary analysis was conducted using MEGA 4 [42].

\section{Amplification of NILT from Atlantic salmon}

Genomic DNA was extracted from an Atlantic salmon liver using a genomic DNA purification system (Promega) following the manufacturer's instructions. The isolated genomic DNA was used as template in a PCR with Ss-pan-NILT primers (Table 1).

The reactions were performed in a total volume of $50 \mu \mathrm{l}$ using $1 \mathrm{U}$ Platinum Taq DNA polymerase High Fidelity (Invitrogen) in the presence of $200 \mathrm{nM}$ primer, $250 \mathrm{ng}$ template, $5 \mu \mathrm{l} 10 \times$ reaction buffer, $2 \mathrm{mM}$ $\mathrm{MgSO}_{4}$ and $200 \mu \mathrm{M}$ dNTPs. The PCR conditions were: initial denaturation at $94^{\circ} \mathrm{C}$ for $1 \mathrm{~min}$ followed by 25 cycles at $94^{\circ} \mathrm{C}$ for $15 \mathrm{~s}, 55^{\circ} \mathrm{C}$ for $15 \mathrm{~s}, 68^{\circ} \mathrm{C}$ for $30 \mathrm{~s}$ and finally one cycle at $68^{\circ} \mathrm{C}$ for $5 \mathrm{~min}$. Atlantic salmon cDNA synthesised from total RNA isolated from head kidney, as previously described [24], was used as template in a PCR with primers designed against NILT. The reactions were performed in a total volume of $50 \mu \mathrm{l}$ using 1.25 U GoTaq DNA polymerase (Promega) in the presence of $200 \mathrm{nM}$ primer, $300 \mathrm{ng}$ template, $10 \mu \mathrm{l}$ $5 \times$ reaction buffer, $1.5 \mathrm{mM} \mathrm{MgCl} 2$ and $200 \mu \mathrm{M}$ dNTPs. The PCR conditions were: initial denaturation at $95^{\circ} \mathrm{C}$ for $2 \mathrm{~min}$ followed by 30 cycles at $95^{\circ} \mathrm{C}$ for $30 \mathrm{~s}, 55^{\circ} \mathrm{C}$ for $30 \mathrm{~s}, 72^{\circ} \mathrm{C}$ for $1 \mathrm{~min}$ and finally one cycle at $72^{\circ} \mathrm{C}$ for $7 \mathrm{~min}$. All PCR products were visualised on 1.5\% agarose gels containing ethidium bromide and ligated into the $\mathrm{pGEM}^{\circ}$ - $\mathrm{T}$ Easy vector (Promega) following the manufacturer's instructions. Plasmids were transformed into chemically competent $E$. coli cells, RapidTran$\mathrm{s}^{\mathrm{Tw}}$ TAM1 (Active Motif) and recombinant clones identified. Plasmid DNA was purified from overnight cell cultures of recombinant clones using a QIAprep Spin Miniprep Kit (Qiagen) following the manufacturer's instructions. Approximately $1 \mu \mathrm{g}$ of purified plasmid DNA was used for sequencing with the vector-specific primers T7 and SP6 by MWG-Biotech (Germany).

\section{Acknowledgements}

We thank the Sequencing Team at the Michael Smith Genome Sciences Centre for sequencing the BAC shotgun library. This work was carried out as part of the CGRASP project founded by Genome Canada and genome BC. This work was supported by a studentship to AEØ from the University of Aberdeen (UK).

\section{Author details}

${ }^{1}$ Scottish Fish Immunology Research Centre, University of Aberdeen, Zoology Building, Tillydrone Avenue, Aberdeen AB24 2TZ, UK. ²Department of

Molecular Biology and Biochemistry, Simon Fraser University, Burnaby, British Columbia, Canada V5A 1S6. ${ }^{3}$ Cell Biology \& Immunology Group, Department of Animal Sciences, Wageningen University, P.O. Box 338, $6700 \mathrm{AH}$ Wageningen, The Netherlands.

\section{Authors' contributions}

AEØ carried out the molecular studies, sequence data analysis, annotations and drafted the manuscript. KPL performed the BAC library screening and contig assembly. SAMM, RJMS, WSD and CJS contributed to the planning, design, and direction of the project. All authors read and approved the final manuscript.

Received: 24 March 2010 Accepted: 9 December 2010 Published: 9 December 2010

\section{References}

1. Magnadóttir B: Innate immunity of fish (overview). Fish Shellfish Immunol 2006, 20(2):137-151.

2. Janeway CA Jr, Medzhitov R: Innate immune recognition. Annu Rev Immunol 2002, 20:197-216.

3. Barclay AN: Membrane proteins with immunoglobulin-like domains - A master superfamily of interaction molecules. Semin Immunol 2003, 15(4):215-233.

4. Wende $H$, Colonna M, Ziegler A, Volz A: Organization of the leukocyte receptor cluster (LRC) on human Chromosome 19q13.4. Mamm Genome 1999, 10(2):154-160.

5. Allcock RJN, Barrow AD, Forbes S, Beck S, Trowsdale J: The human TREM gene cluster at 6p21.1 encodes both activating and inhibitory single lgV domain receptors and includes NKp44. Eur J Immunol 2003, 33(2):567-577.

6. Martin AM, Kulski JK, Witt C, Pontarotti P, Christiansen FT: Leukocyte Ig- like receptor complex (LRC) in mice and men. Trends in Immunology 2002, 23(2):81-88

7. Cantoni C, Ponassi M, Biassoni R, Conte R, Spallarossa A, Moretta A, Moretta L, Bolognesi M, Bordo D: The three-dimensional structure of the 
human NK cell receptor NKp44, a triggering partner in natural cytotoxicity. Structure 2003, 11(6):725-734

8. Bouchon A, Dietrich J, Colonna M: Cutting edge: Inflammatory responses can be triggered by TREM-1, a novel receptor expressed on neutrophils and monocytes. J Immunol 2000, 164(10):4991-4995.

9. Turnbull IR, Gilfillan S, Cella M, Aoshi T, Miller M, Piccio L, Hernandez M, Colonna M: Cutting edge: TREM-2 attenuates macrophage activation. J Immunol 2006, 177(6):3520-3524.

10. Cantoni C, Bottino C, Vitale M, Pessino A, Augugliaro R, Malaspina A, Parolini S, Moretta L, Moretta A, Biassoni R: NKp44, a triggering receptor involved in tumor cell lysis by activated human natural killer cells, is a novel member of the immunoglobulin superfamily. J Exp Med 1999, 189(5):787-795.

11. Radaev S, Kattah M, Rostro B, Colonna M, Sun PD: Crystal structure of the human myeloid cell activating receptor TREM-1. Structure 2003, 11(12):1527-1535.

12. Ravetch JV, Lanier LL: Immune inhibitory receptors. Science 2000, 290(5489):84-89.

13. Campbell KS, Cella M, Carretero M, López-Botet M, Colonna M: Signaling through human killer cell activating receptors triggers tyrosine phosphorylation of an associated protein complex. Eur J Immunol 1998, 28(2):599-609

14. Olcese L, Cambiaggi A, Semenzato G, Bottino C, Moretta A, Vivier E: Human killer cell activatory receptors for MHC class I molecules are included in a multimeric complex expressed by natural killer cells. J Immunol 1997, 158(11):5083-5086.

15. Barrow AD, Trowsdale J: Erratum: You say ITAM and I say ITIM, let's call the whole thing off: The ambiguity of immunoreceptor signalling (European Journal of Immunology (2006) 36 (7)). Eur J Immunol 2006, 36(8):2276..

16. Strong SJ, Mueller MG, Litman RT, Hawke NA, Haire RN, Miracle AL, Rast JP, Amemiya CT, Litman GW: A novel multigene family encodes diversified variable regions. Proc Natl Acad Sci USA 1999, 96(26):15080-15085.

17. Yoder JA, Mueller MG, Wei $\mathrm{S}$, Corliss BC, Prather DM, Willis $\mathrm{T}$, Litman RT, Djeu JY, Litman GW: Immune-type receptor genes in zebrafish share genetic and functional properties with genes encoded by the mammalian leukocyte receptor cluster. Proc Natl Acad Sci USA 2001, 98(12):6771-6776.

18. Hawke NA, Yoder JA, Haire RN, Mueller MG, Litman RT, Miracle AL, Stuge T, Shen L, Miller N, Litman GW: Extraordinary variation in a diversified family of immune-type receptor genes. Proc Natl Acad Sci USA 2001, 98(24):13832-13837.

19. Yoder JA, Mueller MG, Nichols KM, Ristow SS, Thorgaard GH, Ota T, Litman GW: Cloning novel immune-type inhibitory receptors from the rainbow trout, Oncorhynchus mykiss. Immunogenetics 2002, 54(9):662-670.

20. Piyaviriyakul $\mathrm{P}$, Kondo H, Hirono I, Aoki T: A novel immune-type receptor of Japanese flounder (Paralichthys olivaceus) is expressed in both T and B lymphocytes. Fish Shellfish Immunol 2007, 22(5):467-476.

21. Ferraresso S, Kuhl H, Milan M, Ritchie DW, Secombes CJ, Reinhardt R, Bargelloni L: Identification and characterisation of a novel immune-type receptor (NITR) gene cluster in the European sea bass, Dicentrarchus labrax, reveals recurrent gene expansion and diversification by positive selection. Immunogenetics 2009, 61(11-12):773-788.

22. Stet RJ, Hermsen T, Westphal AH, Jukes J, Engelsma M, Lidy Verburg-van Kemenade BM, Dortmans J, Aveiro J, Savelkoul HF: Novel immunoglobulinlike transcripts in teleost fish encode polymorphic receptors with cytoplasmic ITAM or ITIM and a new structural Ig domain similar to the natural cytotoxicity receptor NKp44. Immunogenetics 2005, 57(1-2):77-89.

23. Kock $\mathrm{H}$, Fischer U: A novel immunoglobulin-like transcript from rainbow trout with two Ig-like domains and two isoforms. Mol Immunol 2008, 45(6):1612-1622.

24. Østergaard AE, Martin SAM, Wang T, Stet RJM, Secombes CJ: Rainbow trout (Oncorhynchus mykiss) possess multiple novel immunoglobulin-like transcripts containing either an ITAM or ITIMs. Dev Comp Immunol 2009, 33(4):525-532.

25. Koop BF, Von Schalburg KR, Leong J, Walker N, Lieph R, Cooper GA, Robb A, Beetz-Sargent M, Holt RA, Moore R, Brahmbhatt S, Rosner J, Rexroad CE III, McGowan CR, Davidson WS: A salmonid EST genomic study: Genes, duplications, phylogeny and microarrays. BMC Genomics; BMC Genomics 2008, 9.
26. Ng SHS, Artieri CG, Bosdet IE, Chiu R, Danzmann RG, Davidson WS, Ferguson MM, Fjell CD, Hoyheim B, Jones SJM, De Jong PJ, Koop BF, Krzywinski MI, Lubieniecki K, Marra MA, Mitchell LA, Mathewson C, Osoegawa K, Parisotto SE, Phillips RB, Rise ML, Von Schalburg KR, Schein JE, Shin H, Siddiqui A, Thorsen J, Wye N, Yang G, Zhu B: A physical map of the genome of Atlantic salmon, Salmo salar. Genomics 2005, 86(4):396-404.

27. Burge C, Karlin S: Prediction of complete gene structures in human genomic DNA. J Mol Biol 1997, 268(1):78-94.

28. Altschul SF, Gish W, Miller W, Myers EW, Lipman DJ: Basic local alignment search tool. J MOL BIOL 1990, 215(3):403-410.

29. de Boer JG, Yazawa R, Davidson WS, Koop BF: Bursts and horizontal evolution of DNA transposons in the speciation of pseudotetraploid salmonids. BMC Genomics 2007, 8.

30. Barten R, Torkar M, Haude A, Trowsdale J, Wilson MJ: Divergent and convergent evolution of NK-cell receptors. Trends Immunol 2001, 22(1):52-57.

31. Long EO, Burshtyn DN, Clark WF, Peruzzi M, Rajagopalan S, Rojo S, Wagtmann N, Winter CC: Killer cell inhibitory receptors: Diversity, specificity, and function. Immunol Rev; Immunol Rev 1997, 155:135-144.

32. Alley TL, Cooper MD, Chen M, Kubagawa H: Genomic structure of PIR-B, the inhibitory member of the paired immunoglobulin-like receptor genes in mice. Tissue Antigens 1998, 51(3):224-231.

33. Radley E, Alderton RP, Kelly A, Trowsdale J, Beck S: Genomic organization of HLA-DMA and HLA-DMB. Comparison of the gene organization of al six class II families in the human major histocompatibility complex. J Biol Chem 1994, 269(29):18834-18838.

34. Biassoni R, Cantoni C, Pende D, Sivori S, Parolini S, Vitale M, Bottino C, Moretta A: Human natural killer cell receptors and co-receptors. Immunol Rev 2001, 181:203-214

35. Thorsen J, Zhu B, Frengen E, Osoegawa K, de Jong PJ, Koop BF, Davidson WS, Høyheim B: A highly redundant BAC library of Atlantic salmon (Salmo salar): An important tool for salmon projects. BMC Genomics 2005, 6.

36. Johnstone KA, Lubieniecki KP, Chow W, Phillips RB, Koop BF, Davidson WS: Genomic organization and characterization of two vomeronasal 1 receptor-like genes (ora1 and ora2) in Atlantic salmon Salmo salar. Mar Genomics 2008, 1(1):23-31.

37. Ewing B, Hillier L, Wendl MC, Green P: Base-calling of automated sequencer traces using phred. I. Accuracy assessment. Genome Res; Genome Res 1998, 8(3):175-185.

38. Ewing $B$, Green P: Base-calling of automated sequencer traces using phred. II. Error probabilities. Genome Res; Genome Res 1998, 8(3):186-194.

39. Gordon D, Abajian C, Green P: Consed: A graphical tool for sequence finishing. Genome Res; Genome Res 1998, 8(3):195-202.

40. Bendtsen JD, Nielsen H, Von Heijne G, Brunak S: Improved prediction of signal peptides: SignalP 3.0. J Mol Biol 2004, 340(4):783-795.

41. Thompson JD, Higgins DG, Gibson TJ: CLUSTAL W: Improving the sensitivity of progressive multiple sequence alignment through sequence weighting, position-specific gap penalties and weight matrix choice. NUCLEIC ACIDS RES 1994, 22(22):4673-4680.

42. Kumar S, Tamura K, Nei M: MEGA3: Integrated software for Molecular Evolutionary Genetics Analysis and sequence alignment. Brief Bioinform 2004, 5(2):150-163.

\section{doi:10.1186/1471-2164-11-697}

Cite this article as: Østergaard et al:: Genomic organisation analysis of novel immunoglobulin-like transcripts in Atlantic salmon (Salmo salar) reveals a tightly clustered and multigene family. BMC Genomics 2010 11:697. 